\title{
SWARM ROBOT SOCIAL POTENTIAL FIELDS WITH INTERNAL AGENT DYNAMICS
}

\author{
MABROUK $^{*}$ M. H. and MCINNES ${ }^{* *}$ C.R
}

\begin{abstract}
Swarm robotics is a new and promising approach to the design and control of multiagent robotic systems. In this paper we use a model for a second order non-linear system of self-propelled agents interacting via pair-wise attractive and repulsive potentials. We propose a new potential field method using dynamic agent internal states to successfully solve a reactive path planning problem. The path planning problem cannot be solved using static potential fields due to local minima formation, but can be solved by allowing the agent internal states to manipulate the potential field. Simulation results demonstrate the ability of a single agent to perform reactive problem solving effectively, as well as the ability of a swarm of agents to perform problem solving using the collective behaviour of the entire swarm.
\end{abstract}

\section{KEY WORDS}

Robotics, swarm behaviour, internal agent dynamics, social potential field

$\begin{array}{ll}\text { NOMENCLATURE } \\ C_{a i} & \text { Amplitude of attraction potential of the } \mathrm{i}^{\text {th }} \text { agent } \\ C_{g i} & \text { Amplitude of goal total potential affecting the } \mathrm{i}^{\text {th }} \text { agent } \\ C_{o i} & \text { Amplitude of obstacle potential affecting } \mathrm{i}^{\text {th }} \text { agent } \\ C_{r i} & \text { Amplitude of repulsive potential of the } \mathrm{i}^{\text {th }} \text { agent } \\ l_{a i} & \text { Range of attractive potential for the } \mathrm{i}^{\text {th }} \text { agent } \\ l_{g i} & \text { Range of goal potential affecting the } \mathrm{i}^{\text {th }} \text { agent } \\ l_{o i} & \text { Range of obstacle potential affecting the } \mathrm{i}^{\text {th }} \text { agent } \\ I_{r i} & \text { Range of repulsive potential of the } \mathrm{i}^{\text {th }} \text { agent } \\ m_{i} & \text { Mass of the } \mathrm{i}^{\text {th }} \text { agent } \\ N_{p} & \text { Number of agents } \\ Q_{i} & \text { The } \mathrm{i}^{\text {th }} \text { agent internal dynamic function } \\ \mathbf{r}_{i g} & \text { Goal }-\mathrm{i}^{\text {th }} \text { agent position vector } \\ \mathbf{r}_{i j} & \mathrm{i}^{\text {th }}-\mathrm{j}^{\text {th }} \text { agent position vector } \\ \mathbf{r}_{i o} & \text { Obstacle }-\mathrm{i}^{\text {th }} \text { agent position vector } \\ \mathbf{r}_{c} & \text { Swarm centre position vector }\end{array}$

${ }^{*}$ PhD research student, Dept. of Mechanical Engineering, University of Strathclyde, Glasgow, UK.

${ }^{* *}$ Professor, Dept. of Mechanical Engineering, University of Strathclyde, Glasgow, UK. 


$\begin{array}{ll}U\left(\mathbf{r}_{i}\right) & \text { Generalized Morse potential } \\ U_{g}\left(\mathbf{r}_{g}\right) & \text { Goal potential field } \\ U_{i j}\left(\mathbf{r}_{i j}\right) & \mathrm{i}^{\text {th }}-\mathrm{j}^{\text {th }} \text { agent interaction potential } \\ \mathbf{v}_{c} & \text { Swarm centre velocity vector } \\ \mathbf{v}_{i} & \mathrm{i}^{\text {th }} \text { agent velocity vector } \\ \alpha & \text { Self-accelerating force coefficient } \\ \beta & \text { Friction self-decelerating force coefficient }\end{array}$

\section{INTRODUCTION}

The design and control of artificial swarms has become a topic of growing interest. Swarm robotics has a range of applications in both civilian and military fields from space and subsea exploration to the deployment of teams of interacting artificial agents in disposal systems [1]. Several researchers have proposed novel methods of generating control algorithms for individual agents, including learning and evolutionary algorithms $[2,3]$. In addition, the design and control of agents to accomplish specific collective goals has drawn considerable interest in recent years [4]. True artificial swarm design has been largely developed through two main approaches. The first is based on a set of practical, algorithmic approaches [5 -8]. In contrast, the second method is based on artificial physics [9]. Both methods have been applied to teams of autonomous agents.

The study of agent-based systems begins with a definition of the term agent [10, 11]. An individual agent may be programmed to be fully autonomous, but its abilities may be limited according to resource and physical constraints. On the other hand, swarms of self-organizing agents that exchange information may have a greater functionality than the individual members. Natural examples of interacting swarms of agents can be found in ants, bees, birds and schools of fish in the way that they create complex patterns with new and useful group properties $[12,13]$. In recent years, an understanding of the operating principles of natural swarms has proven to be a useful tool for the intelligent design and control of artificial robotic agents [14, 15]. Many swarming systems have been investigated and complex behaviour such as phase transitions have been observed [15-18].

Swarming robotic systems are often modelled as a two-dimensional collection of point agents in which members may interact with one another through attractiverepulsive pair-wise interactions. Specific choices of potential field lead agents to selforganize into coherent patterns $[15,18-20]$. More recently, swarm stabilization or collapse with increasing constituent number in different zones of a so-called $\mathrm{H}$-stability diagram, shown in Fig. 1, has been predicted [16, 17, 21]. Using tools from statistical mechanics [22] connections between the so-called $H$-stable nature of the interaction potential and resulting aggregating patterns have been found. Virtual leaders [1, 21] and structural potential functions $[23,24]$ have also been introduced to provide provable group behaviour to ensure vehicles can avoid obstacles or form desired patterns. The actual realizations of self-propelled vehicles interacting according to virtual Morse potentials have been reported in the robotics literature $[1,25]$. These prior studies assume that the free parameters of the potential field are fixed a priori. While in here we assume the parameters to be internal states for each agent through which the agent can manipulate the potential field. The dynamics of these internal states is defined through set of first order differential equations. 


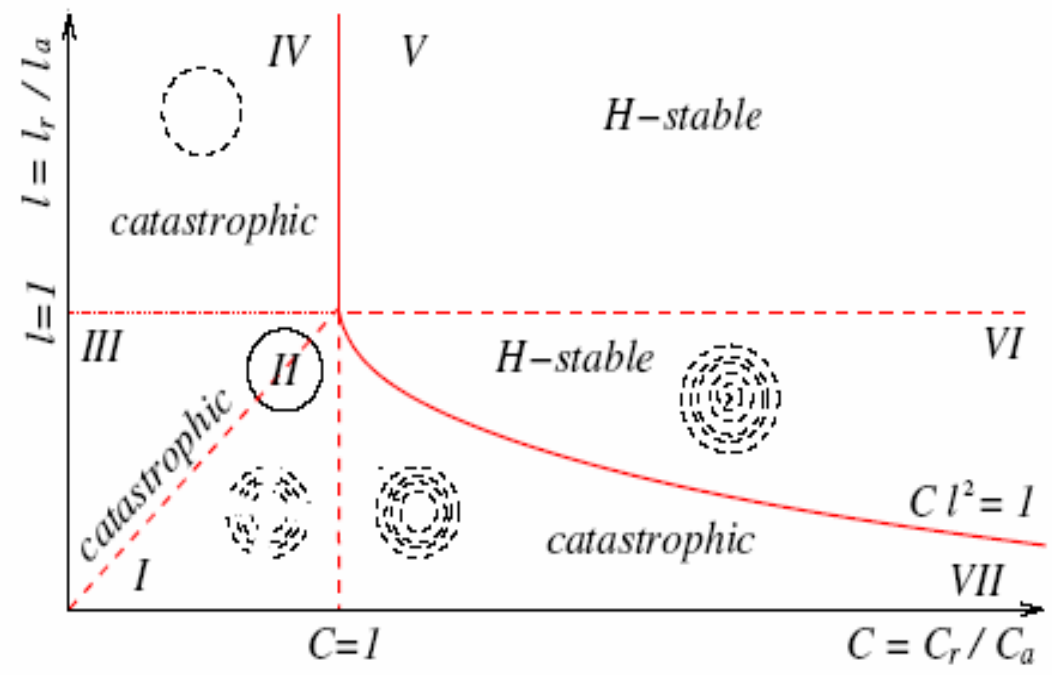

Fig.1. $\mathrm{H}$-stability phase diagram of the Morse potential. Catastrophic and stable behaviors are predicted as a function of the parameter ratios $\left(I_{r} / l_{a}\right)$ and $\left(C_{r} / C_{a}\right)$ [adapted from reference 16$]$

In this paper we use a simple model of driven, self-propelled agents which also experience some dissipative frictional force. The model consists of $N_{p}$ particles with mass $m_{i}$, position $\mathbf{r}_{i}$ and relative distance $\left|\mathbf{r}_{i j}\right|$ between the $i^{\text {th }}$ and $j^{\text {th }}$ agent. A selfaccelerating force with coefficient $\alpha$ is introduced and to prevent the particles from reaching large speeds, a dissipative friction force with coefficient $\beta$ is added [16]. The agents interact by means of a two-body generalized Morse potential, which decays exponentially at large distances and represents a realistic description of natural and artificial swarming agents. The equations of motion of the $N_{\mathrm{p}}$ agents are defined as:

$$
\begin{aligned}
& \frac{d \mathbf{r}_{i}}{d t}=\mathbf{v}_{i} \\
& m_{i} \frac{d \mathbf{v}_{i}}{d t}=\left(\alpha-\beta\left|\mathbf{v}_{i}\right|^{2}\right) \mathbf{v}_{i}-\nabla U\left(\mathbf{r}_{i}\right) \\
& U\left(\mathbf{r}_{i}\right)=\sum_{j \neq i}^{N_{p}} C_{r} \exp \left(-\left|\mathbf{r}_{i}-\mathbf{r}_{j}\right| / l_{r}\right)-C_{a} \exp \left(-\left|\mathbf{r}_{i}-\mathbf{r}_{j}\right| / l_{a}\right)
\end{aligned}
$$

This model has been used in other studies such as those reported in [16-18, 25]. The potential is characterized by attractive and repulsive potential fields of strength $C_{a}$ and $C_{r}$ with ranges $I_{a}$ and $I_{r}$ respectively. From Eq. (2), it can be seen the velocity of the agents will reach $\left|\mathbf{v}_{i}\right|=\sqrt{\alpha / \beta}$ asymptotically, where the term $\alpha-\beta\left|\mathbf{v}_{i}\right|^{2}$ vanishes. For simplicity we will consider unit mass agents. 


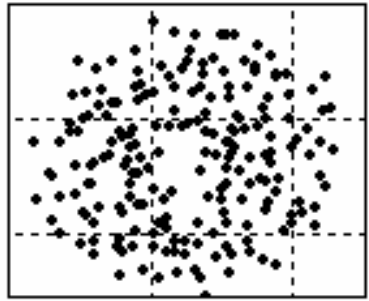

a

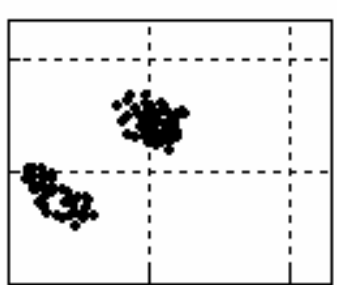

b

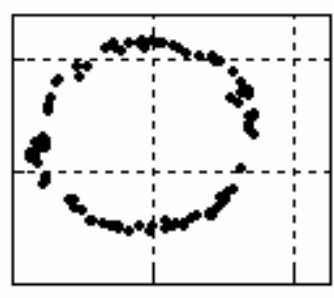

C

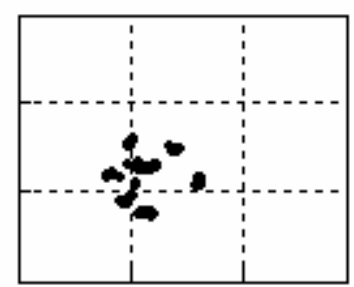

d

Fig.2. Patterns of swarm of agents in different $H$-stability regions and with different interaction parameters. (a) Vortex, region $\mathrm{VII}, N_{p}=200, I_{r}=0.5, l_{a}=2, C_{r}=1, C_{a}=0.5$, $\alpha=1.6, \beta=0.5$ (b) Clumps, region I, $N p=100, I_{r}=0.5, l_{a}=1, C_{r}=0.6, C_{a}=1, \alpha=1, \beta=0.5$ (c) Ring, region II, $N_{\mathrm{p}}=100$, Ir=0.5, la=1, $C_{r}=0.5, C_{a}=1, \alpha=1, \beta=0.5$ (d) Ring clumping, region III, $N_{p}=100, l_{r}=1.2, l_{a}=1, C_{r}=0.6, C_{a}=1, \alpha=1, \beta=0.5$

We have developed a simulation code (using an Adams-Bashforth numerical integration method) to predict the swarm behaviour in different zones of the $H$-stability diagram, shown in Fig. 1, and using different parameters to test the integrity of the simulation according to the predicted behaviour of the swarm patterns. Fig. 2 shows results which match those in the literature $[16,17]$. The agents were given random initial positions and velocities as well as fixed, identical values of the potential free parameters.

\section{PROBLEM DEFINITION}

In recent years new assumptions about the architecture needed for intelligence have emerged. These approaches attempt to emulate natural, rather than artificial intelligence and are based on, or at least inspired by, biology. In an attempt to build a control system for autonomous agents, Balkenius [26] presented a general architecture for behaviour-based control. He proposed a number of architectural principles which make it possible to combine reactive control with problem solving in a coherent way. He used the term behaviour to denote the system internal to the agent that is responsible for the externally observed behaviour.

The problem of local minima (trapped states), shown in Fig. 3, was discussed by Balkenius [26]. The reactive problem for an agent, or swarm of agents, attracted to a goal point at position $G$ can be defined such that an artificial potential field at $G$ induces motion towards the goal. When the agent, or swarm of agents, moves towards the goal the velocity of each individual agent rises, and the agents translate to the goal along the gradient of the potential field. However, in order prevent collision with a static obstacle, an additional repulsive potential field is required. These two potential fields are then superimposed to form a global potential field which describes the workspace of the problem. In general however, a local minimum may form due to the superposition of the goal potential and that of the obstacles, resulting in the agent, or swarm of agents, becoming trapped in a state other than the goal $G$. 
Considering this problem, the entire swarm, or part of the swarm will be trapped at the

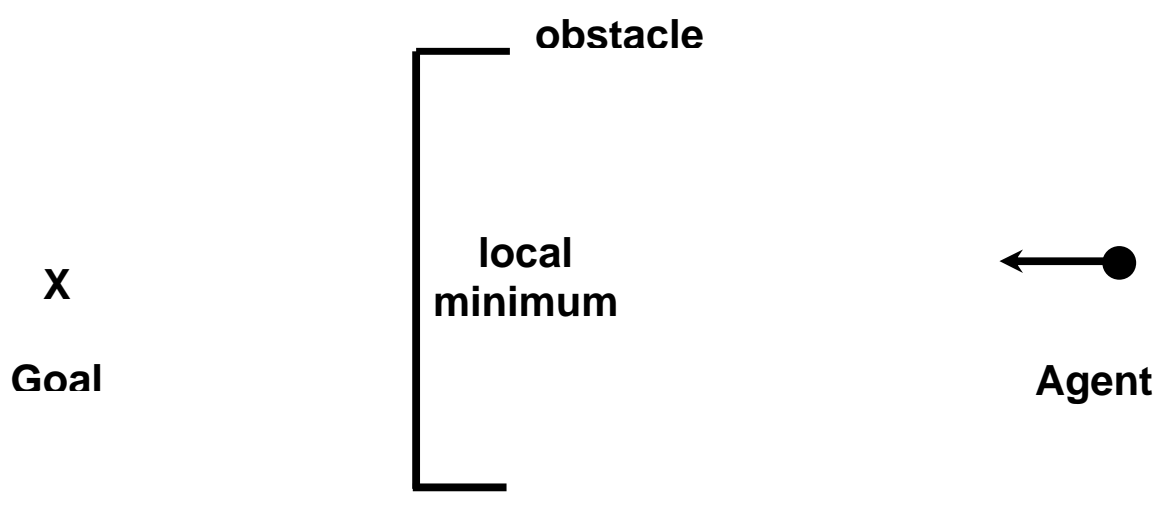

Fig.3. Classical reactive problem for one agent

obstacle since the agents trapped inside the obstacle will experience two virtual forces; the first force is the attraction to the goal while the other will be the repulsion from the obstacle. Moreover in most cases there will be no opportunity for the swarm members to escape from the local minimum due to the pair-wise interaction potential - particularly when the goal potential is of large amplitude. This problem motivates the use of the collective swarm behaviour to avoid such trapping in local minima and leads to two specific sub-problems:

(a) A swarm of self-propelled agents are trapped in a local minimum - when one agent escapes, it will become a leader for the rest of the trapped agents

(b) A single agent is trapped in a local minimum - it must be then be able to escape depending only on its own internal states to manipulate the potential field

\section{SWARM LEADER}

In order to investigate the concept of the swarm leader, and how it affects the global swarm behaviour, the motion of a swarm whose individuals experience attraction to a goal point will now be considered. Assuming that the agents are maneuvering such that $\left|\mathbf{v}_{i}\right| \sim \sqrt{\alpha / \beta}$, the agents' equation of motion is defined from Eq. (2) as:

$$
\ddot{\mathbf{r}}_{i}=-\nabla U\left(\mathbf{r}_{i}\right)-\nabla U_{g}\left(\mathbf{r}_{i g}\right)
$$

where $U_{g}$ is the goal potential field. This can then be expanded to form:

$$
\ddot{\mathbf{r}}_{i}=-\nabla_{i} \sum_{j \neq i}^{N_{p}} U_{i j}\left(\mathbf{r}_{i j}\right)-\nabla_{i} U_{g}\left(\mathbf{r}_{i g}\right)
$$


Noting that $\nabla_{i} U_{i j}\left(\mathbf{r}_{i j}\right)=U_{i j}^{\prime}\left(\mathbf{r}_{i j}\right) \hat{\mathbf{r}}_{i j}$ and $\nabla_{i} U_{g}\left(\mathbf{r}_{i g}\right)=U^{\prime}{ }_{i g}\left(\mathbf{r}_{i g}\right) \hat{\mathbf{r}}_{i g}$ it can be seen that:

$$
\ddot{\mathbf{r}}_{i}=-\sum_{j \neq 1}^{N_{p}} U^{\prime}{ }_{i j}\left(\mathbf{r}_{i j}\right) \hat{\mathbf{r}}_{i j}-U^{\prime}{ }_{i g}\left(\mathbf{r}_{i g}\right) \cdot \hat{\mathbf{r}}_{i g}
$$

where, $U_{i j}^{\prime}\left(\mathbf{r}_{i j}\right)=\partial U_{i j} / \partial \mathbf{r}_{i j}$. Considering now the aggregate motion of the swarm through the swarm centre, defined such that $\ddot{\mathbf{r}}_{C}=1 / N p \sum_{i}^{N p} \ddot{\mathbf{r}}_{i}$, substituting from Eq. (6) will yield:

$$
\ddot{\mathbf{r}}_{c}=-\frac{1}{N_{p}} \sum_{i}^{N_{p}} \sum_{j \neq i}^{N_{p}} U^{\prime}{ }_{i j}\left(\mathbf{r}_{i j}\right) \hat{\mathbf{r}}_{i j}-\frac{1}{N_{p}} \sum_{i}^{N_{p}} U^{\prime}{ }_{i g}\left(\mathbf{r}_{i g}\right) \hat{\mathbf{r}}_{i g}
$$

Then, since $\hat{\mathbf{r}}_{i j}=-\hat{\mathbf{r}}_{j i}$, the double summation in Eq. (7) will cancel to yield:

$$
\ddot{\mathbf{r}}_{c}=-\frac{1}{N_{p}} \sum_{i}^{N_{p}} U_{i g}^{\prime}\left(\mathbf{r}_{i g}\right) \hat{\mathbf{r}}_{i g}
$$

Therefore, it can be seen that the swarm center accelerates toward the goal $G$. In principle, the agents can therefore be attracted to any swarm member which is considered to be a temporary goal, if it has a large attractive potential parameter $C_{a}$.

Consider $N_{p}$ identical self-propelled agents which are trapped behind a barrier that consists of $N_{o}$ identical obstacle points as shown in Fig. 4. $G$ is a goal point which has an attraction potential of low interaction range $l_{g}$. The challenge in this situation (problem (a)) is that the agents are stuck inside a trap whose only exit is located away from the goal position. In this situation, we define a condition that if one of the agents finds its way through the exit, through random motion, it will gain a higher attraction potential coefficient $C_{a}$, higher attraction potential range $l_{a}$ and higher dissipation coefficient $\beta$ to lower its speed, increasing the opportunity for the rest of the agents to follow. These conditions now make any succeeding agent a temporary goal for the rest of the agents and the swarm center therefore accelerates to the leader position, leading them out of the trap. Subsequently the swarm is then attracted to the goal.

The simulation result in Fig. 4 shows that after escaping from the trap the swarm is attracted to the goal point $G$ and so the entire swarm escapes from the trap and moves towards the goal as required. We now consider problem (b) which is how an individual will be able to escape from a local minimum depending only on its own self potential. This will raise another question of how an agent can sense that it is trapped at a location other than the goal. 


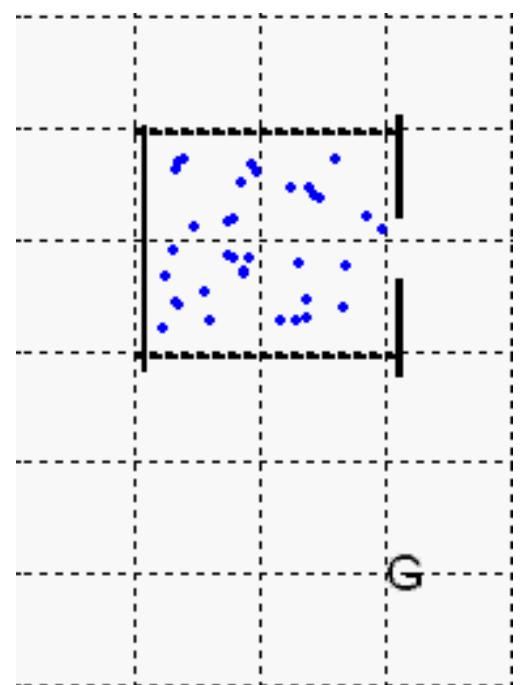

(1)

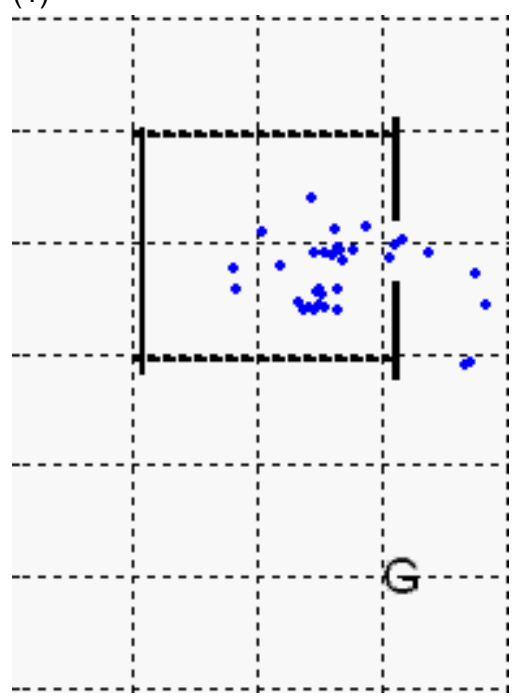

(4)

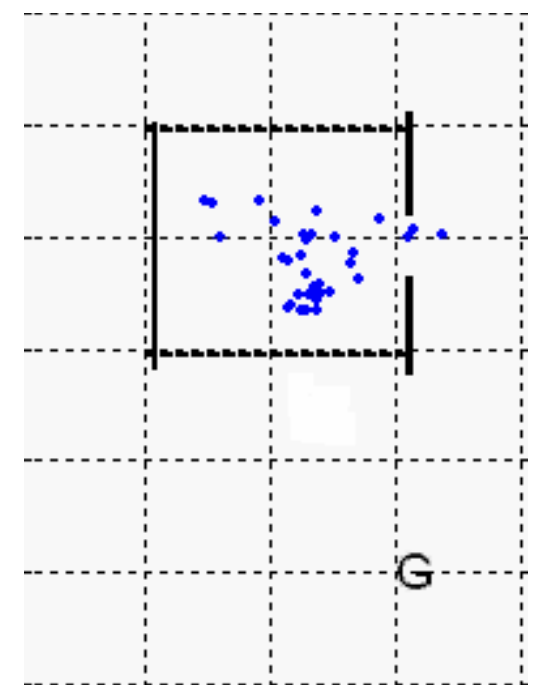

$(2)$

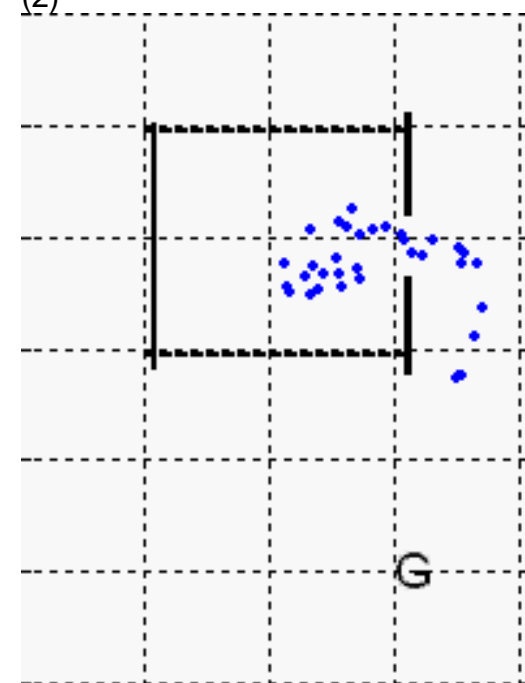

(5)

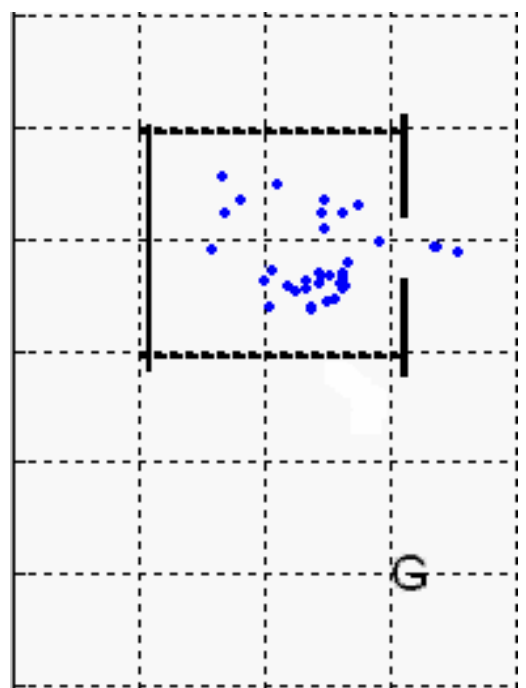

(3)

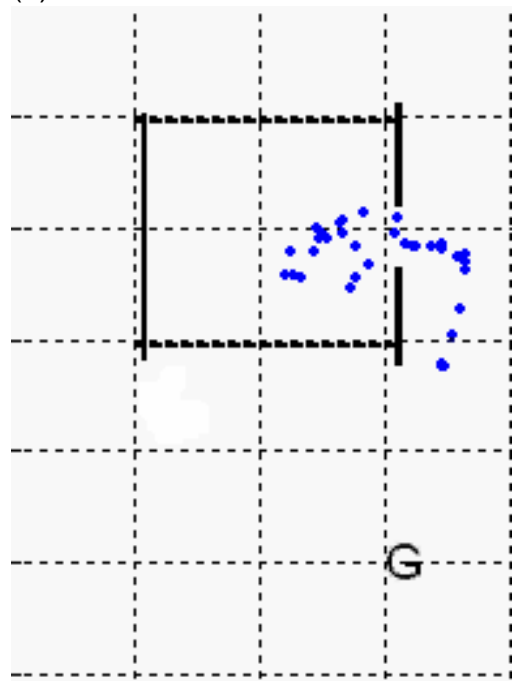

(6)

Fig.4. Escape from local minima using a swarm leader

\section{SWARM SQUEEZE EFFECT}

Escape from complex workspaces can be seen in many natural systems in which the system consists of a number of agents enclosed in a trap. An example is a system of gas molecules which are enclosed in a single-exit container while the molecules experience a change in their state, due to a rise in temperature for example. Let $(a, b)$ be two points each of which represents two adjacent regions $(A, B)$ respectively. Assume that both have the same potential and let the center of a swarm of agents be at point (a) enclosed in a trap of obstacles. Due to some change of state, the region $A$ is then forced to become a region of maximum potential while the adjacent region $B$ remains unchanged. 


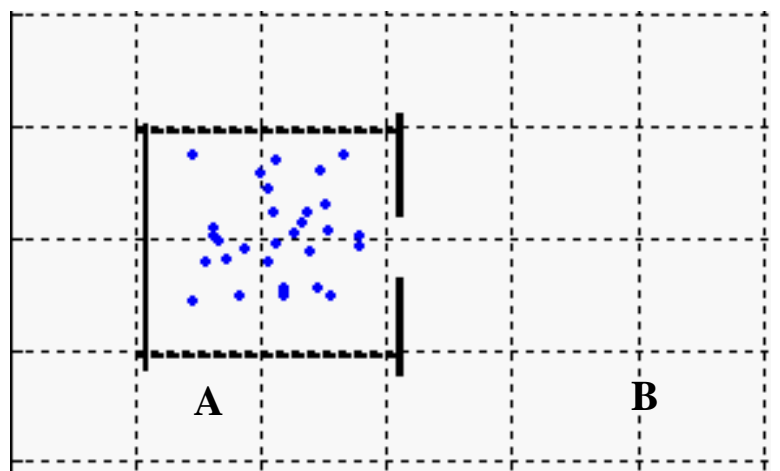

(1)

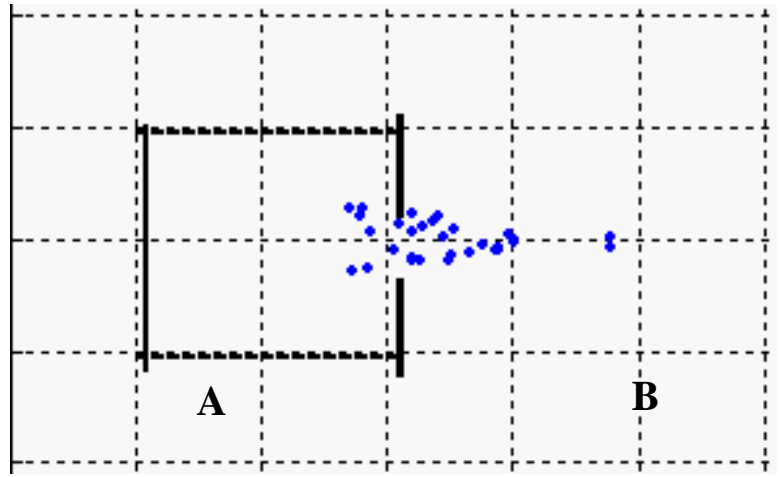

(3)

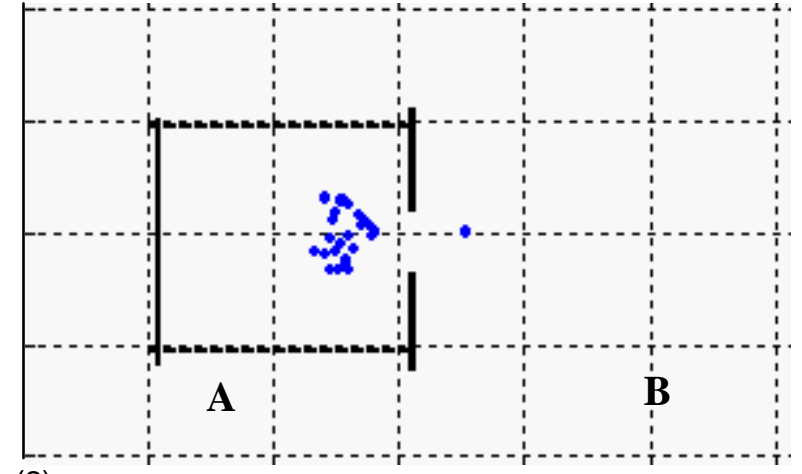

(2)

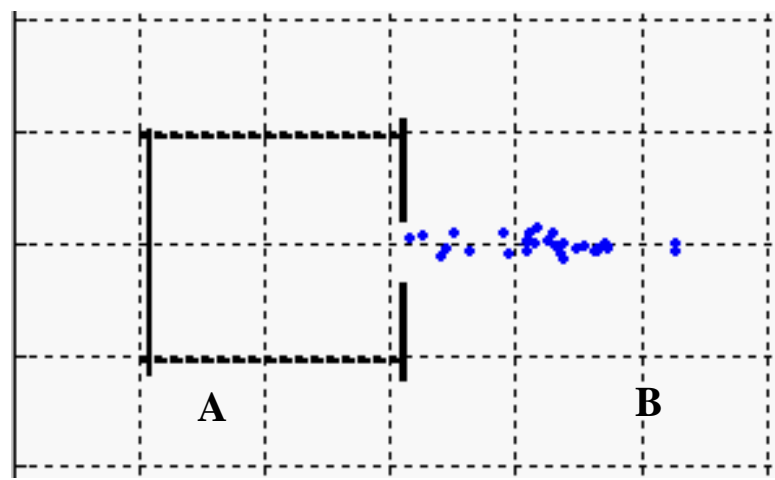

(4)

Fig.5. Squeeze effect

Therefore, the swarm center moves from (a) towards (b). The change of the internal state of the system simply changes the trap region from a local minimum into a region of maximum potential from which all the agents are emitted as if squeezed out [22], as shown in the simulation result Fig. 5. Here the repulsive interaction potential of each agent has been increased, leading both to an increase in repulsion between agents and between the walls of the trap. The use of agent internal states (potential field free parameters) will now be considered as a means of allowing agents to manipulate the potential field in which they are maneuvering in order to escape from local minima.

\section{AGENT INTERNAL STATE MODEL}

The previous section has demonstrated that a change in the internal state of the agents can lead to escape from a trap (manipulating a local minimum into a local maximum). This concept will now be used for a single agent maneuvering towards a goal in a potential field which contains a local minimum. The agent internal states (potential field free parameters) will now be defined through a set of differential equations which will allow the agent to manipulate the potential field in which it is maneuvering.

For a fixed obstacle, the repulsion potential range affecting the $i^{\text {th }}$ agent $\left(I_{o i}\right)$ can be represented as a function of an obstacle constant $\left(I_{0}\right)$, which characterizes the physical nature of the obstacle, and the particle repulsion potential range $\left(I_{r i}\right)$ which characterizes the agent internal state. The attraction potential range of the goal affecting the $i^{\text {th }}$ agent 
$\left(I_{g i}\right)$ can also be represented as a function of a goal constant $\left(I_{g}\right)$, which characterizes the physical nature of the goal, and the particle attraction potential range $\left(I_{a i}\right)$ which characterizes the agent internal state such that:

$$
\begin{aligned}
& C_{o i}=C_{o} \\
& l_{o i}=l_{o}+l_{r i} \\
& C_{g i}=C_{g} \\
& l_{g i}=l_{g}+l_{a i}
\end{aligned}
$$

When an agent approaches an obstacle it suffers an elastic collision which pushes the agent away from the goal. The goal then attracts the agent back and the agent will never attempt to maneuver around the obstacle simply because it never knows it's trapped.

A function $Q_{i}$ is now defined, inspired from the learning by reward or punishment $[27,28]$, and applied to the agent and its ability to perceive the environment in which it exists. The internal dynamic function is defined as the change of the modulus of the agent's velocity measured in some interval of time interval. If the agent is repulsed from an obstacle $Q_{i}$ will have a negative value which is punishment, because the agent is moving away from the goal. If the agent is moving towards the goal $Q_{i}$ will have a positive value which is the reward as the agent senses it is moving to the goal. The following set of differential equations are used to express the internal states of the agents defined as:

$$
\begin{aligned}
& \dot{C}_{r i}=A_{r} \exp \left(-Q_{i}\right)-\lambda_{r} C_{r i} \\
& \dot{l}_{r i}=B_{r} \exp \left(-Q_{i}\right)-\lambda_{r} l_{r i} \\
& \dot{C}_{a i}=A_{a} \exp \left(Q_{i}\right)-\lambda_{a} C_{a i} \\
& \dot{l}_{a i}=B_{a} \exp \left(Q_{i}\right)-\lambda_{a} l_{a i} \\
& \dot{\beta}=A_{\beta} \exp \left(-Q_{i}\right)-\lambda_{\beta} \beta
\end{aligned}
$$

Equations (13-16) express the repulsion amplitude and range and the attraction amplitude and range of the $i^{\text {th }}$ agent, according to the use of the function $Q_{i}$. For $Q_{i}<0$ it can be seen that the agent will experience repulsion (section 4), leading to motion away from obstacles, while for $Q_{i}>0$ the agent experiences attraction (section 3) and motion towards the goal (or other lead agent). Moreover, Eq. (17) ensures a smooth maneuver around the obstacle by slowing the agents which are moving away from the obstacle. The damping terms in Eq. (13-16) ensure that the deviation of the agent internal state is minimized in the absence of external forcing from the function $Q_{i}$. The benefit of the function $Q_{i}$ is that when the agents are repelled $\left(Q_{i}<0\right), I_{o i}$ takes high a value which turns the workspace in the neighborhood of the obstacles into a zone of maximum potential. This then leads to escape from the local minima (section 4), with the potential 
field relaxing after escape due to the damping terms in the differential equations for the internal states.

The simulation shows that using the swarm model, Eqs. (1-3), along with the dynamic internal states, Eqs. (13-17), the potential field around the obstacle is converted from a local minimum into a local maximum. This will ensure the agent is pushed from the obstacles and will maneuver around them. After the agent is squeezed away it will follow the gradient of the potential field around the obstacles and approach the goal $G$.

First, the case of an agent with fixed internal states will be considered. Here the free parameters describing the potential field, and so the potential field itself, are constant. The contour map shown in Fig. 6 is static and so the agent becomes trapped in the local minimum of the potential field. This is typical of conventional implementations of the artificial potential field method to path planning problems.

For dynamical internal states the contour map in Fig. 7 shows that the agent enters the local minimum, and when repelled $\left(Q_{1}<0\right)$ the repulsion potential of the agent increases in a way that converts the obstacle to be a zone of maximum potential to the agent. As the agent escapes from the local minimum the potential field relaxes due to the damping terms in Eqs. (13-16). The goal potential field then drags the agent away from the obstacle zone and defines a gradient path that the agent follows directly to the goal. The comparison between the contour maps in Fig. 6 and Fig. 7 shows clearly the effect of using the function $Q_{i}$ and internal state dynamics to solve the reactive problem effectively.

Finally, the simulation results shown in Fig. 8 show the use of the swarm leader concept. The agents at the front of the swarm are repelled $\left(Q_{i}<0\right)$ so that their internal states are modified such that their own repulsive potential increases. This in turn pushes the rest of the swarm away from the obstacle. The agents which are then moving towards to goal $\left(Q_{>}>0\right)$ act as leaders and so attract the remainder of the swarm.
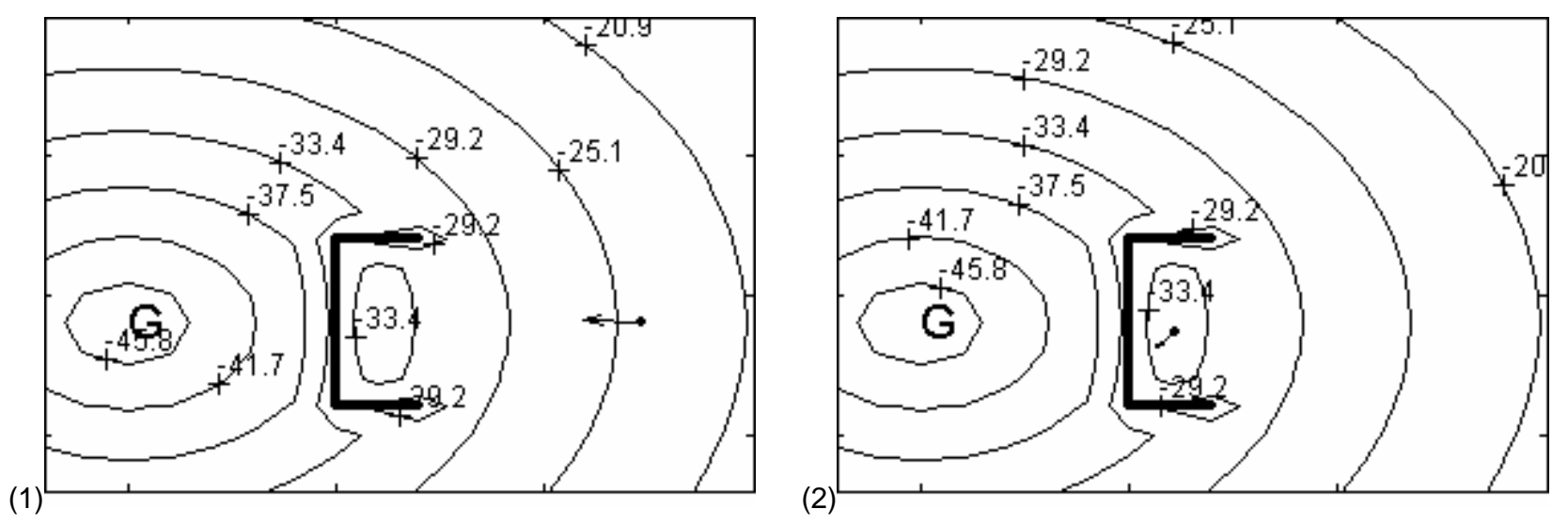

Fig. 6. Behaviour of a conventional agent with fixed internal states 

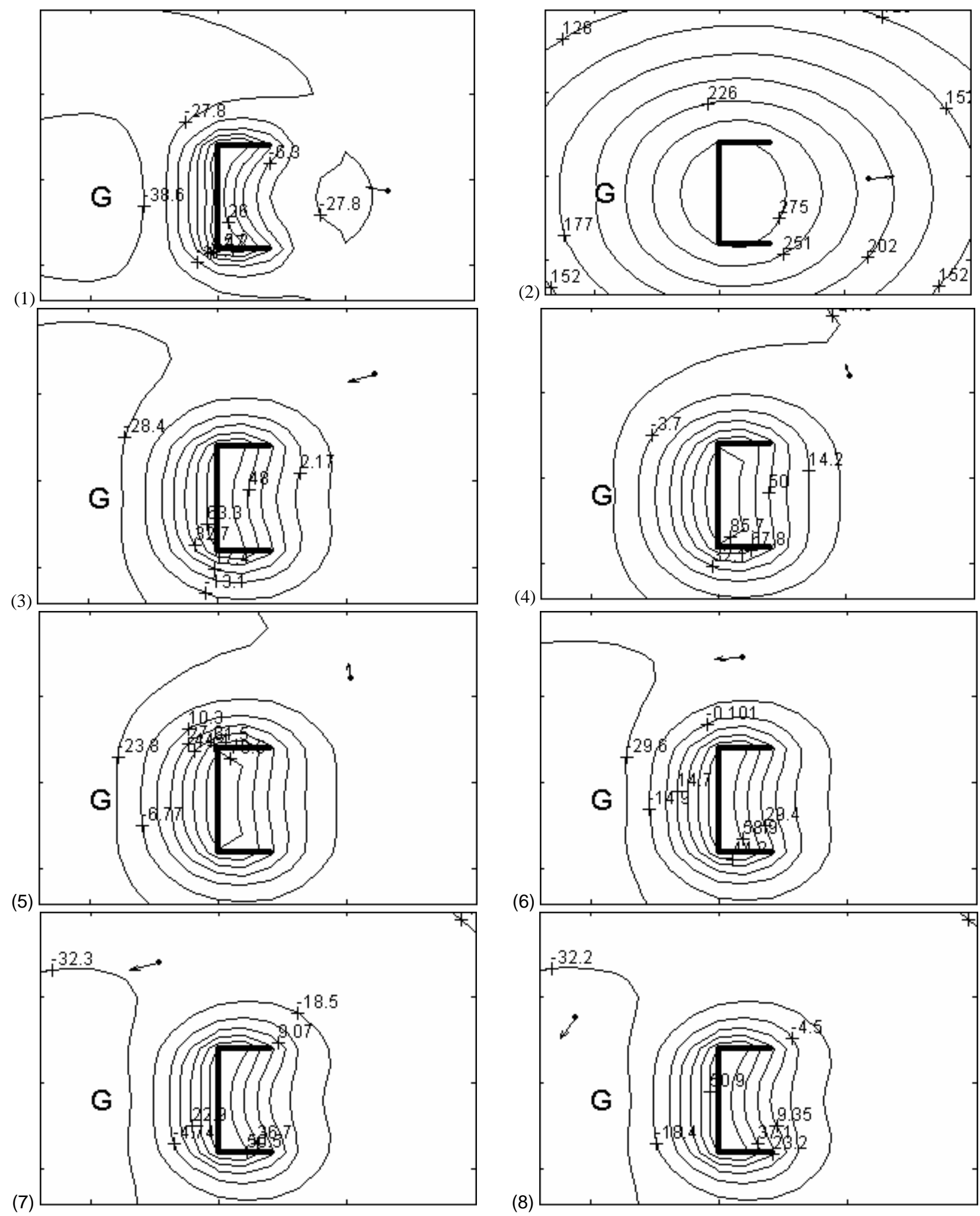

(4)

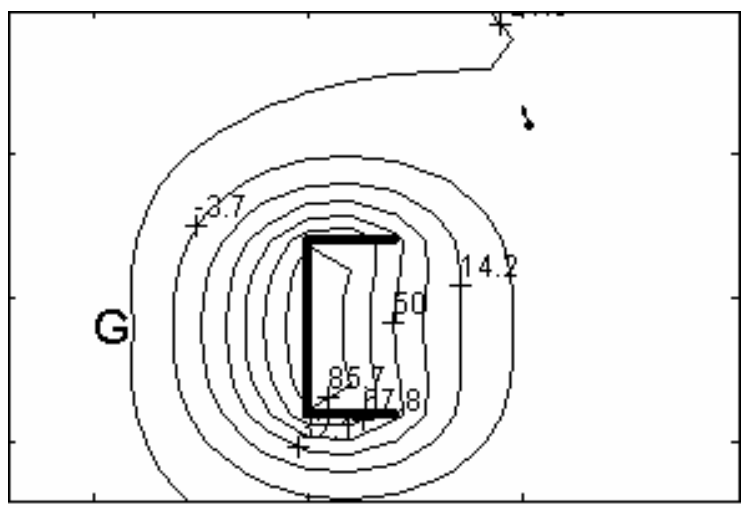

(6)

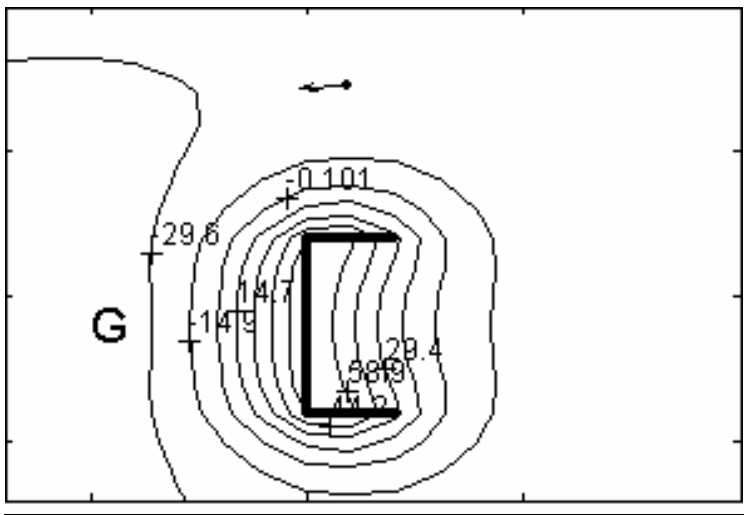

(8)

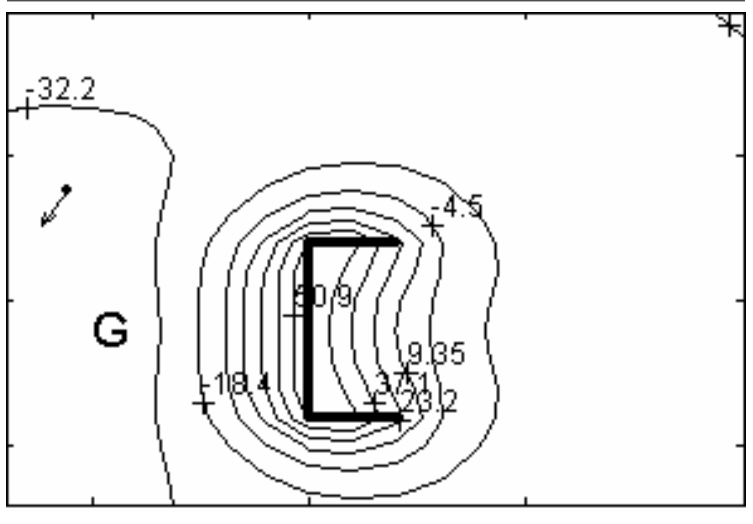

Fig. 7. Behaviour of an agent using the internal state model 


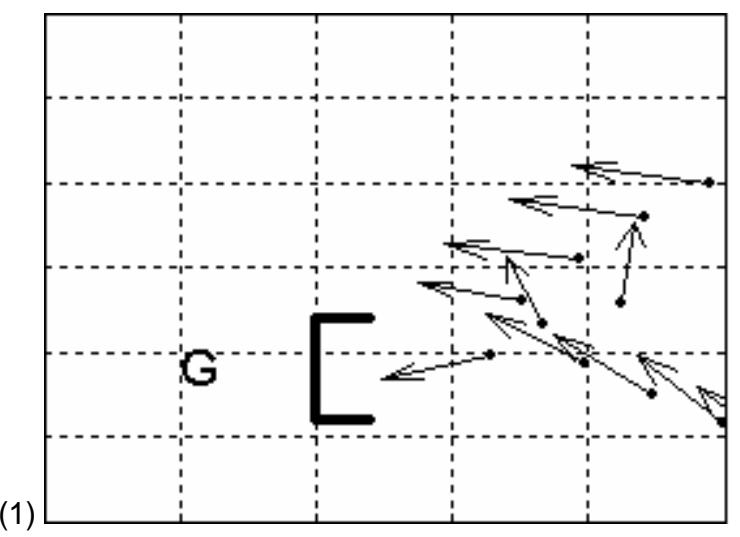

(3)

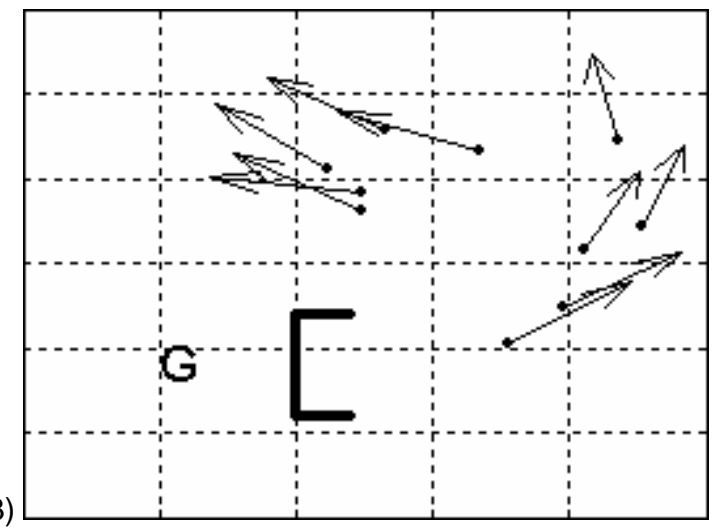

(5)

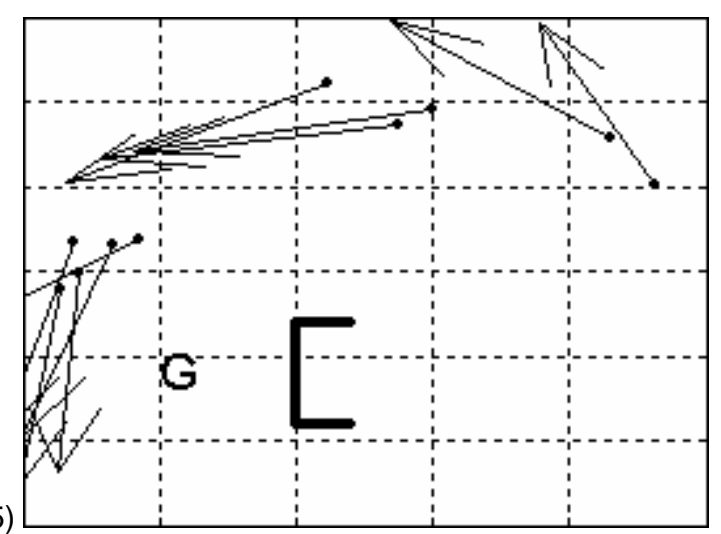

(2)

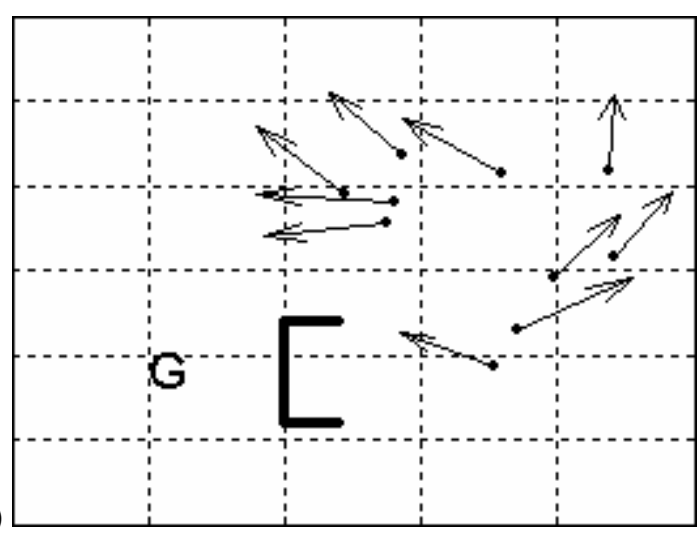

(4)

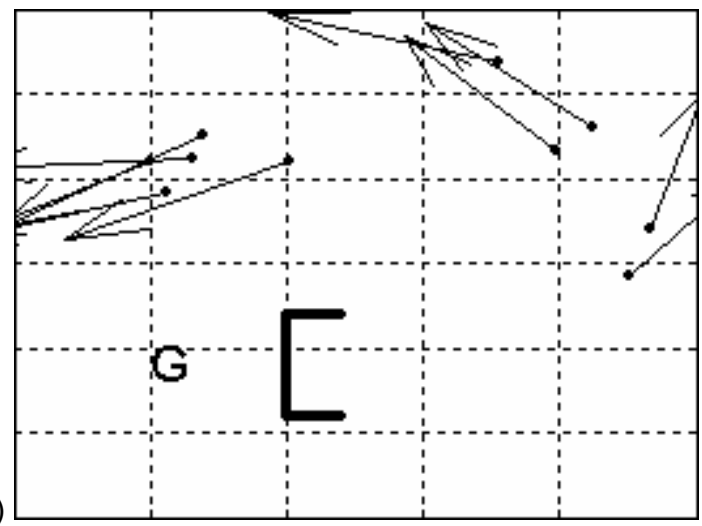

(6)

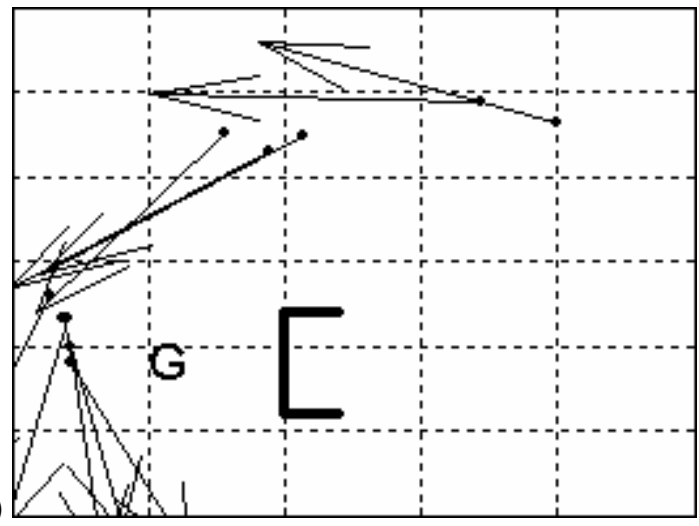

Fig. 8. Behaviour of a swarm using the internal state model

\section{CONCLUSIONS}

A new potential field method has been presented which uses the concept of an agent internal state to allow agents to manipulate the potential field in which they maneuver. The method allows a swarm of agents to escape from and maneuver around a local minimum in the potential field to reach a goal. Rather than moving in a static potential field, the agents are able to manipulate the potential according to their estimation of whether they are moving towards or away from the goal. Using the function $Q_{i}$ along with the use of the concepts of the swarm leader and the squeeze effect, simulation results show that a swarm of agents can indeed escape from a local minimum. 


\section{REFERENCES}

[1] N. E. Leonard and E. Fiorelli, "Virtual Leaders, Artificial Potentials, and Coordinated Control of Groups", in Proc. Conf. Decision Contr., Orlando, FL, pp. 2968-2973, 2001.

[2] Harvey I. "Evolutionary Robotics and SAGA: The Case for Hill Crawling and Tournament Selection", Proceedings of Artificial Life 3, Langton C., ed. Reading, Massachusetts: Addison Wesley, 299-326, 1994.

[3] Mataric M. and Cliff D., "Challenges in Evolving Controllers for Physical Robots", Robotics and Autonomous Systems, 19 (1): 67-83, 1996.

[4] E W Justh, P S Krishnaprasad, "Steering Laws and Continuum Models for Planar Formations", Proceedings of the 42nd IEEE International Conference on Decision and Control 3609-3614,2003

[5] Kelly I. and Keating D, "Faster Learning of Control Parameters Through Sharing Experiences of Autonomous Mobile Robots", International Journal of Systems Science. 29(7), 783-793, 1998.

[6] Mataric M., "Designing and Understanding Adaptive Group Behaviour", Adaptive Behavior, 4 (1): 51-80, 1995.

[7] Mataric M., "Issues and Approaches in the Design of Collective Autonomous Agents", Robotics and Autonomous Systems, 16 (2-4): 321-331, 1995.

[8] Mataric M., "Behaviour-Based Control: Examples From Navigation, Learning, and Group Behaviour", Journal of Experimental and Theoretical Artificial Intelligence, 9 (2-3): 323-336, 1997.

[9] Spears W., and Gordon D., "Using Artificial Physics to Control Agent", Proceedings of IEEE International Conference on Information, Intelligence, and Systems (ICIIS'99), November, 1999.

[10] Maes P., "Modelling Adaptive Autonomous Agents", Artif. Life, 1(1-2):135 - 162, 1994.

[11] Beer R. "Dynamical Systems Perspective an Agent-Environment Interaction", Artificial Intelligence, 72: 173-215, 1995.

[12] S. Camazine, J. L. Deneubourg, N. R. Franks, J. Sneyd, G. Theraulaz, and E. Bonabeau, "Self Organization in Biological Systems", Princeton University Press, Princeton, NJ, 2003.

[13] J K Parrish, L Edelstein-Keshet, "Complexity, Pattern, and Evolutionary Tradeoffs in Animal Aggregation", Science 284:99-101, 1999.

[14] E. Bonabeau, M. Dorigo, and G. Theraulaz, "Swarm Intelligence: From Natural to Artificial Systems", Oxford University Press, New York, 1999.

[15] V. Gazi and K. Passino, "Stability Analysis of Swarms", in IEEE Trans. Autom. Contr. Vol. 48, pp. 692-697, 2003.

[16] M. R. D'Orsogna, Y. L. Chuang, A. L. Bertozzi and L. Chayes, "Self-Propelled Agents With Soft-Core Interactions: Patterns, Stability, and Collapse", Phys. Rev. Lett., Vol. 96, 104302, 2006.

[17] M. R. D'Orsogna, Y. Chuang, A. Bertozzi, and L. Chayes "Pattern Formation, Stability and Collapse in 2D Driven Particle Systems", pages 103-115, edited by

A. Bulsara and S. Baglio (Springer-Verlag, Berlin Heidelberg, 2006).

[18] H. Levine, W. J. Rappel, and I. Cohen "Self-Organization in Systems of SelfPropelled Agents", Phys. Rev. E, Vol. 63, pp. 017101, 2000. 
[19] V. Gazi and K. Passino, "A Class of Attractions/Repulsion Functions for Stable Swarm Aggregations", in Proc. Conf. Decision Contr., Las Vegas, NV, pp. 28422847, 2002.

[20] V. Gazi and K. Passino, "Stability Analysis of Social Foraging Swarms: Combined Effects of Attractant/Repellent Profiles", in Prof. Conf. Decision Contr., Las Vegas, NV, pp. 2848-2853, 2002.

[21] Yao-Li Chuang , Yuan R. Huang , Maria R. D'Orsogna1 and Andrea L. Bertozzi1, "Multi-Vehicle Flocking: Scalability of Cooperative Control Algorithms Using Pairwise Potential", accepted in the 2007 IEEE Int. Conf. on Robotics and Automation.

[22] D. Ruelle, "Statistical Mechanics, Rigorous Results", W. A. Benjamin Inc, New York, NY, 1969

[23] R. Olfati-Saber and R. M. Murray, "Distributed Cooperative Control of Multiple Vehicle Formations Using Structural Potential Functions", in $15^{\text {th }}$ IFAC World Congress, Barcelona, Spain, 2002.

[24] R. Olfati-Saber, "Flocking for Multi-Agent Dynamic Systems: Algorithms and Theory", IEEE Trans. on Autom. Contr., Vol. 51, 2006.

[25] B. Q. Nguyen, Y. L. Chuang, D. Tung, C. Hsieh, Z. Jin, L. Shi, D. Marthaler, A. L. Bertozzi, and R. M. Murray, "Virtual Attractive Repulsive Potentials for Cooperative Control of Second Order Dynamic Vehicles on the Caltech MVWT", in Proc. American Contr. Conf., Portland, OR, pp. 1084-1089, 2005.

[26] Balkenius, C. "Natural Intelligence for Autonomous Agents", (extended version), Lund University Cognitive Studies, 29,1994.

[27] Mackintosh, N. J., "Conditioning and Associative Learning", Oxford: Oxford University Press, 1983.

[28] Lieberman, D. A., "Learning: Behaviour and Cognition", Belmont, CA: Wadsworth Publishing Company,1990. 